\title{
Imaging the communicating structures on oocyte through high-resolution spinning-disc microscope
}

\author{
Yan Zhang \\ China Agricultural University \\ Ye Wang \\ China Agricultural University \\ Hua Zhang ( $\nabla$ huazhang@cau.edu.cn ) \\ China Agricultural University
}

\section{Method Article}

Keywords: oocyte, granulosa cell, high-resolution 3D imaging, oocyte microvilli

Posted Date: March 16th, 2021

DOl: https://doi.org/10.21203/rs.3.pex-1381/v1

License: (1) This work is licensed under a Creative Commons Attribution 4.0 International License.

Read Full License 


\section{Abstract}

The communications between oocyte and granulosa cells are essential for ovarian folliculogenesis in mammals, and the communicating structures in the ZP had been widely reported. However, there was lack of direct approach to visualize these structures in a 3D model with a high-resolution. Here, we present a protocol to image the structures which derived from oocytes by combining endogenous fluorescent mouse models with a high-resolution spinning disc-confocal system in details

\section{Introduction}

As a conserved structure in the zona pellucida, communicating microvilli or transzonal projections (TZPs) have been well documented to exist between oocytes and granulosa cells (GCs) in ovarian follicles of different species including the mammals. However, the origin and the detail structure of these communicating structures are elusive since lacking of cell specific labeling method and high-resolution imaging approach. To visualize the microvilli structures and distinguish oocytic cellular origin during follicle development, we used an $\mathrm{mT} / \mathrm{mG}$ reporter mouse model[1] in which membrane-localized GFP $(\mathrm{mG})$ is inducible expressed in Cre-positive cells by crossing with oocyte-specific Gdf9-Cre mice[2]. We established a 3D high-resolution imaging system to distinguish the derivation of microvilli by drawing the outlines of the cellular membrane with $\mathrm{mG}$.

\section{Reagents}

Mice

Gdf9-Cre (Gdf9-icre, JAX 011062)

$R_{O S A}{ }^{m T / m G}$ mice $(m T / m G ;$ JAX 007576)

Sample preparation and culturing:

MEM a, nucleosides, GlutaMAX ${ }^{\text {TM }}$ Supplement (Gibco, 32-571-036)

Fetal Bovine Serum (Gibco, 10-099-141)

ITS Liquid Media Supplement (100x) (Sigma-Aldrich, 13146)

$100 \mathrm{U} / \mathrm{ml}$ penicillin and $100 \mu \mathrm{g} / \mathrm{ml}$ streptomycin (Thermo Fisher Scientific, 15140122)

PBS (Gibco, 20-012-027)

Mineral oil (Sigma-Aldrich, M8410) 


\section{Equipment}

Sterile $1 \mathrm{~mL}$ syringe (BD, 300841)

Omnican U-40 Insulin syringe (B.Braun, 9161635S)

Mouth pipette for handling and denudation of oocytes

Leica bright field dissection microscope (MZ10)

$35 \mathrm{~mm}$ culture dish (Nest, 706001)

35 mm glass bottom dish (Cellvis, D35-14-1.5-N)

Andor Dragonfly 502 spinning-disk confocal with living cell workstation (Okolab)

\section{Procedure}

1. Generation of Gdf9-Cre;mTmG mice:

(1) To generate the Gdf9-Cre;mTmG mouse models, adult healthy Gdf9-Cre males were crossed with $m T / m G$ female mouse in $1: 2$.

(2) DNA of mouse tail extracted and genotyped to identify the Gdf9-Cre,mTmG female before postnatal day (PD) 7.

Gdf9-Cre primers: TCTGATGAAGTCAGGAAGAACC

GAGATGTCCTTCACTCTGATTC

$m T / m G$ primers: CTCTGCTGCCTCCTGGCTTCT

CGAGGCGGATCACAAGCAATA

TCAATGGGCGGGGGTCGTT

\section{Preparation of culture medium:}

Medium a (MEMa; Gibco) was supplied with 10\% FBS (Gibco), 1\% insulin-transferrin-selenium (ITS; Sigma-Aldrich) and $0.1 \%$ penicillin and streptomycin (Thermo Fisher Scientific), and was pre-warmed to $37^{\circ} \mathrm{C}$ before using. 


\section{Collecting the denuded oocytes:}

(1) Ovary was collected from Gdf9-Cre,mTmG females at PD 35.

(2) Remove the ovarian bursa carefully by sterile $1 \mathrm{~mL}$ syringe in cold PBS buffer.

(3) By tearing the ovaries with Omnican U-40 insulin syringe, denuded oocytes at GV stages were collected in the culture medium. Only the healthy oocytes with an average diameter around $65 \mu \mathrm{m}$ were collected for the further experiments.

(4) Washing the collected oocytes three times with $~ 50 \mu \mathrm{L}$ culture medium microdroplet by mouth pipette gently.

*Tips: Transferring the oocytes gently to guarantee the integrity of ZP.

4. Imaging the cellular membrane structure on living oocytes:

(1) Transfer around 10 oocytes to the culture medium microdroplet $(\sim 20 \mu \mathrm{L})$ in $35 \mathrm{~mm}$ glass bottom dish.

(2) Covered the microdroplet with $1.5 \mathrm{~mL}$ mineral oil (Sigma-Aldrich).

(3) Photographed in a living cell workstation (Okolab) at $37^{\circ} \mathrm{C}, 5 \% \mathrm{CO}_{2}$ by Andor Dragonfly 502 spinning-disc confocal with following index. In details, images were acquired by an Andor Dragonfly 502 spinning-disc confocal microscope equipped with a 63x, 1.40 N.A. oil objective (Leica HC PL APO), a scientific complementary metal-oxide semiconductor (sCMOS) camera (Andor Zyla 4.2), and 488-nm $(\mathrm{mG})$ and 568-nm (mT) lines of the Andor Integrated Laser Engine (ILE) system with a spinning-disc confocal scan head (Andor Dragonfly 500). The oocytes were acquired through z-step mode with the index. In detail, images were acquired with laser 488-nm around 10-20\%, laser 568-nm around 15-25\%, exposure time 100-200 ms and Z-step 0.5-0.7 $\mu \mathrm{m}$ for 25-35 $\mu \mathrm{m}$ by Fusion 2.1 software.

(https://andor.oxinst.com/products/dragonfly\#fusion).

(4) After acquisition, videos or single time-point images were processed by Image J (http://rsbweb.nih.gov/ij/) for projection of all z-stacks and merged color channels.

(5) To finally detect the Oo-Mv on oocyte, the rotary 3D video was processed by Imaris (https://imaris.oxinst.com/) software.

*Tips: The index of imaging might be changed with the situations of microscope such as the environment temperature and humidity. 


\section{Troubleshooting}

The index of imaging might be changed with the situations of microscope such as the environment temperature and humidity.

\section{Time Taken}

About 2-4 h (except for breeding mice)

\section{References}

[1] Zheng, W. et al. Two classes of ovarian primordial follicles exhibit distinct developmental dynamics and physiological functions. Hum. Mol. Genet. 23, 920-928 (2014).

[2] Lan, Z.-J., Xu, X. \& Cooney, A. J. Differential oocyte-specific expression of Cre recombinase activity in GDF-9-iCre, Zp3cre, and Msx2Cre transgenic mice. Biol. Reprod. 71, 1469-1474 (2004).

[3] Mogessie, B. \& Schuh, M. Actin protects mammalian eggs against chromosome segregation errors. Science 357, eaal1647 (2017)

\section{Figures}




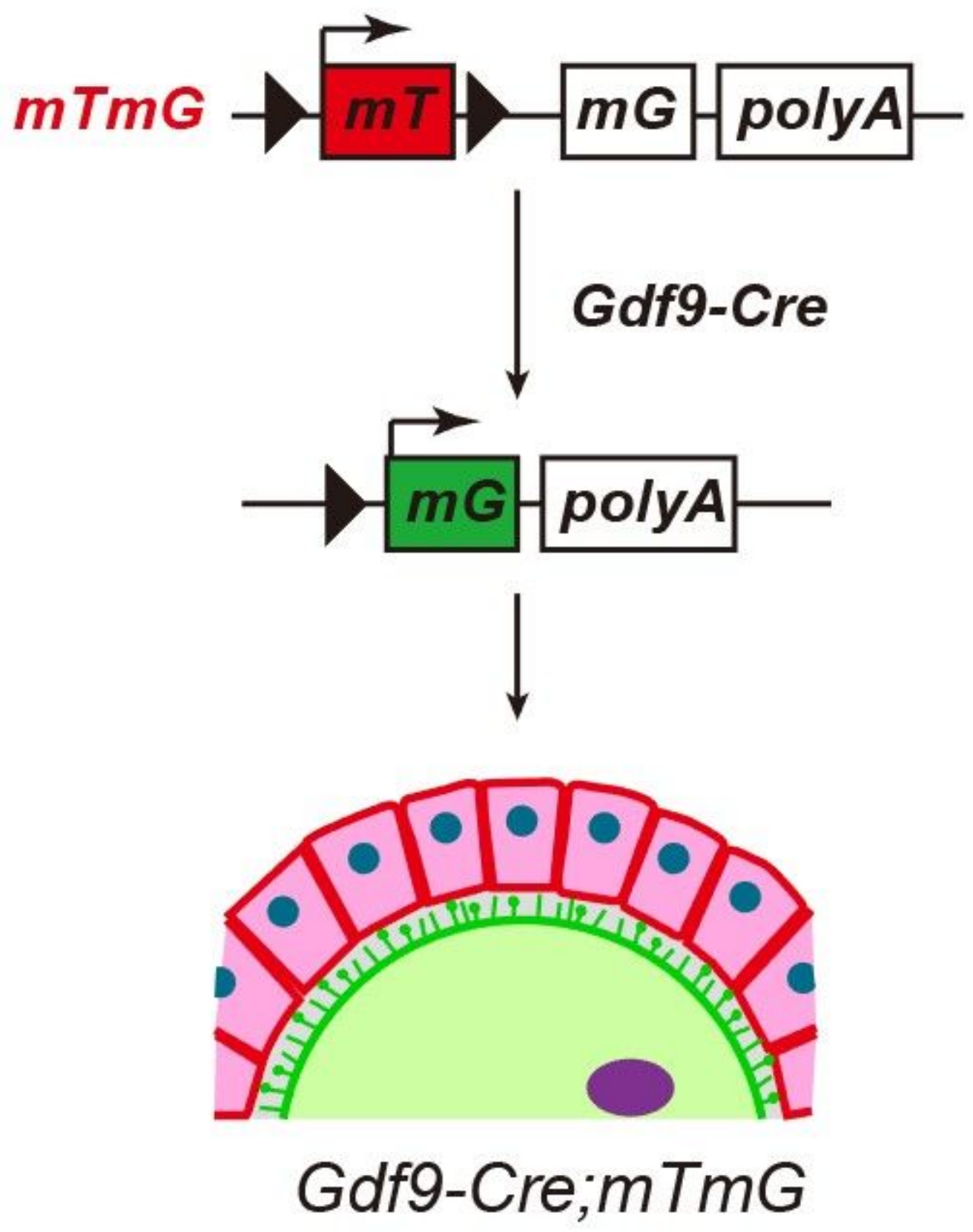

Figure 1

Illustration of the strategy to label the oocyte microvilli by Gdf9-Cre;mTmG 
Gdf9-Cre;mTmG Living oocyte (Oo-mv)
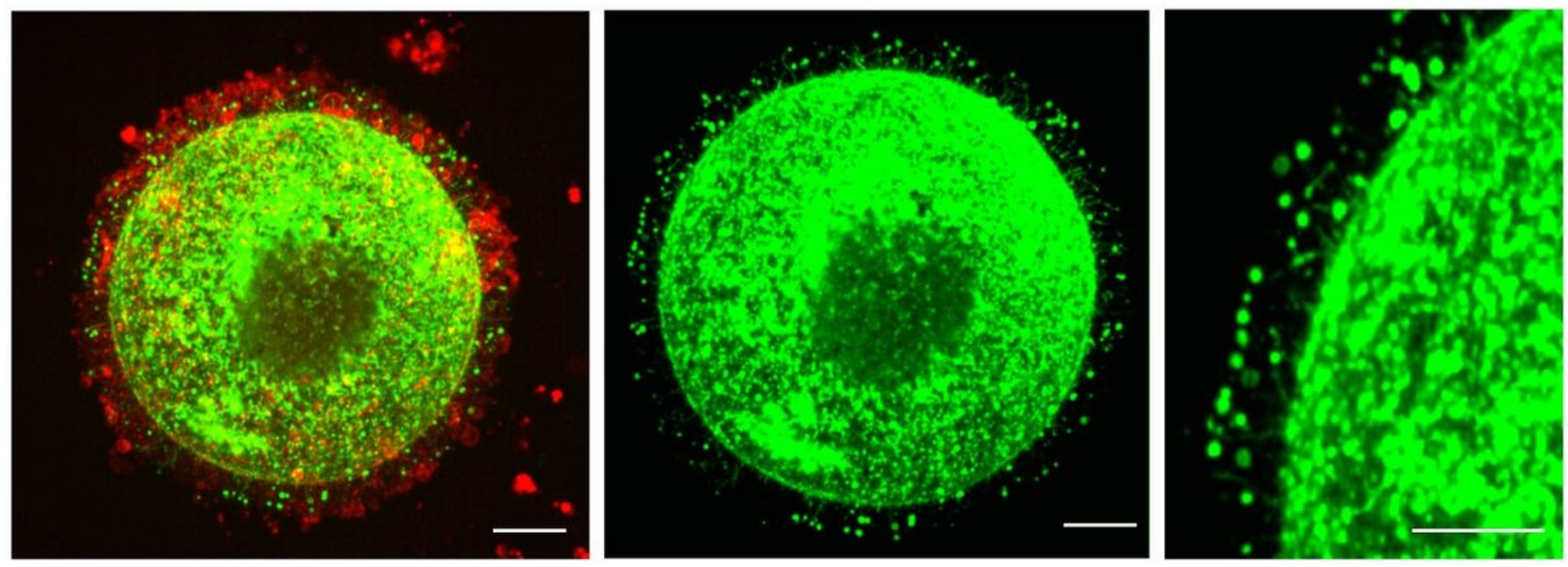

Figure 2

Image of the oocyte microvilli in the ZP by live Gdf9-Cre;mTmG oocyte 